\title{
La coopération éducative internationale
}

Axe de formation ou métier à part entière

Daniel Denis

\section{CpenEdition}

\section{Journals}

Édition électronique

URL : https://journals.openedition.org/ries/4038

DOI : $10.4000 /$ ries. 4038

ISSN : 2261-4265

Éditeur

France Education international

Édition imprimée

Date de publication : 1 juin 1995

Pagination : 73-80

ISSN : 1254-4590

Référence électronique

Daniel Denis, «La coopération éducative internationale », Revue internationale d'éducation de Sèvres [En ligne], 06 | 1995, mis en ligne le 01 janvier 2015, consulté le 08 décembre 2022. URL : http:// journals.openedition.org/ries/4038; DOI : https://doi.org/10.4000/ries.4038

Ce document a été généré automatiquement le 29 septembre 2020

Tous droits réservés 


\title{
La coopération éducative internationale
}

\author{
Axe de formation ou métier à part entière
}

\author{
Daniel Denis
}

\section{NOTE DE L'ÉDITEUR}

Propos recueillis par Chantal Paisant.

Chantal PAISANT : Vous avez conduit pendant une douzaine d'années, de 1980 à 1992', une expérience de formation de futurs instituteurs à la «coopération éducative internationale ». II faudra préciser ce que peut recouvrir cette notion, en formation initiale, mais d'abord: qu'est-ce qui a motivé l'expérience, au départ?

Daniel Denis : Elle est avant tout le fait d'une équipe, j'y insiste tout de suite, une équipe de huit puis douze formateurs qui partageaient une même conception de l'école publique et des enjeux de la formation d'instituteurs à la fin $\mathrm{du} \mathrm{xx}^{\mathrm{e}}$ siècle : en des termes nuancés pour chacun d'entre nous, il nous semblait évident que l'internationalisation économique et politique mettait en cause les repères transmis par l'école et notamment l'idée de l'État-nation. Nous avons considéré que l'école publique devait prendre en compte cette instigation majeure pour exercer une fonction pertinente dans la formation des citoyens ayant prise sur le monde contemporain. La composante personnelle - une expérience de l'étranger inscrite dans la biographie de chacun d'entre nous - a joué un rôle dans cette prise de conscience qu'un pays comme la France ne correspond plus à l'image que son historiographie, ses grands mythes fondateurs continuent de donner en dépit du réel. Mais un autre facteur nous a déterminés : ce sont les implications locales de l'internationalisation, l'immigration. C'est cela au départ qui nous a mobilisés : partir des réalités de la classe, dans un département comme celui des Hauts-de-Seine et essayer de les éclairer autrement. Nos premiers projets ont trouvé leur légitimité dans les réalités du département. 
Chantal paisant : Est-ce que vous pouvez donner tout de suite des exemples de ces premiers projets?

Daniel Denis : Nous avons commencé à travailler dans les communes du nord du département, où les classes peuvent grouper quatre à six nationalités différentes, en nous demandant quelles solutions étaient apportées à ce problème de la diversité ethnique et culturelle dans les banlieues des grands centres urbains par les AngloSaxons. Nous avons travaillé trois mois dans des classes de la banlieue de San Francisco, pendant la campagne électorale des commissions scolaires, en cherchant à comparer les implications de la politique américaine, fondée de longue date sur l'autonomie des collectivités et celle de la centralisation à la française. Le second projet, mené en parallèle avec un autre groupe de normaliens, nous a conduits à Haïti, pour tâcher de comprendre le rôle de l'éducation dans les processus de développement.

Chantal paisant : Vous venez d'évoquer des séjours d'étude de trois mois. Comment de tels projets s'intégraient-ils au cursus de formation sans entrer en concurrence avec les autres composantes de la formation au métier d'instituteur?

Daniel Denis : L'équipe des formateurs, une fois encore, est déterminante pour mettre en mouvement vingt à cinquante normaliens ${ }^{2}$ qui vont travailler sur un même projet pendant deux ans. C'est une équipe interdisciplinaire où chacun cède des heures pour le projet (nous disions « l'impôt sur les disciplines »). A la fois parce qu'on considère que chaque discipline a son rôle à jouer dans la formation de l'enseignant et dans la mesure où toutes concourent à l'enjeu central. Le projet international est l'axe central interdisciplinaire de la formation, sachant que chaque discipline garde, au-delà du projet, sa dimension propre et son autonomie. On utilisait l'expression " marcher sur deux pieds » : premier pied, structurer la formation autour d'un objectif central (en l'occurrence, développer l'intelligence de l'incidence de l'internationalisation sur les problèmes éducatifs) ; second pied, savoir faire la classe. Il s'agit bien de former à un métier et non de faire $d u$ "Sciences-Po pour instituteurs». Il faut souligner ici les méfiances et les malentendus qu'ont suscité les voyages d'étude (dans les tracts syndicaux, Haïti devenait Tahiti, on formait les enseignants à la promenade !). Il ne s'agissait pourtant pas d'une fuite hors de l'école mais d'un travail dans et pour l'école. Avant qu'il y ait des zones d'éducation prioritaires (ZEP) et des projets d'établissement, nos étudiants travaillaient dans les écoles du département pour y conduire des travaux en rapport avec leur thème. Ils ne partaient pas à l'étranger sans un investissement fort dans l'école de rattachement: le savoir sur l'autre est investi dans la pratique professionnelle.

Chantal paISANT : Vous insistez sur cet ancrage dans les écoles. Mais quel était le rôle des classes ? Champs d'observation ? Laboratoires ? Acteurs?

Daniel Denis : Il y a eu une évolution. Au départ, en 1980-1981, on a travaillé dans des écoles, puis à partir de 1984-1985, dans des classes qui se prêtaient à nos projets. Enfin, en 1989, ce sont les classes elles-mêmes qui se sont engagées dans des échanges internationaux à leur niveau : les normaliens n'étaient plus les inventeurs de projets d'échanges, ils étaient intégrés à des classes qui devenaient les moteurs des échanges. On incitait les instituteurs à définir des thèmes pour les appariements. C'est là la première et principale mutation. 
CHANTAL PAISANT : À partir de 1985-1986, il y a eu une seconde évolution, géopolitique, qui pendant deux cycles de formation, deux fois deux ans, a réorienté les projets vers les enjeux éducatifs de la construction européenne. Comment reliez-vous les deux évolutions?

Daniel Denis : Elles se conçoivent dialectiquement. Le fait que les échanges se fassent au niveau des enfants et que l'étude critique des relations intra-européennes porte sur l'école est révélateur de ce qui devenait une préoccupation collective - la démocratie scolaire - dans un contexte où la construction européenne, l'Acte unique, n'était pas encore, en 1985-1986, l'objet d'un débat public. Quelle Europe voulions-nous? Un espace de prospérité indifférent au reste du monde? Une citadelle construite contre l'extra-européen et en particulier contre l'islam? Les enjeux de la formation dépassent le clivage entre mondialistes et pro-européens. Nous pensions qu'il fallait, qu'il faut, travailler avec les enfants à la construction d'une Europe ouverte, c'est-à-dire capable d'analyser l'histoire de son rapport à l'extra-européen. Former une équipe, c'est aussi concilier des perceptions différentes et il n'est pas sûr que nous soyons allés jusqu'au bout des implications de cette idée fondamentale qui constitue le point de passage obligé d'une future citoyenneté internationale : l'histoire commune à l'Europe est celle de ses conflits; depuis le $\mathrm{xVI}^{\mathrm{e}}$ siècle, l'Europe est un condominium de rivalités pour la conquête du monde. On ne lutte pas contre les stéréotypes sans jouer avec eux pour faire comprendre comment Français et Anglais, par exemple, se perçoivent à travers une histoire conflictuelle. Tout le travail pédagogique est là : comprendre ce qui trame nos attitudes nationales face à l'autre et en quoi ce rapport à l'autre construit notre identité nationale; détramer nos rivalités. Les enfants français et anglais qui ont travaillé pendant deux ans sur la Tapisserie de Bayeux et se sont présentés mutuellement leur théâtre de Guillaume le Conquérant, avec un superbe travail en arts plastiques, ont-ils compris cela? On peut l'espérer. En tout cas c'est la direction à explorer car les propos de type bruxellois, basés sur un postulat très irénique ne peuvent que susciter des réactions hyper-nationalistes. Nous avions une pleine conscience des enjeux du métier d'enseignant et en même temps la volonté de soulever cet énorme problème.

Chantal paISANT : Vous évoquiez à l'instant les défiances des syndicats d'enseignants eux-mêmes à l'égard des premiers projets avec San Francisco et Haïti : comment l'institution a-t-elle accueilli les projets suivants?

Daniel Denis : J'ai parlé d'équipe déjà à deux reprises. Je dois maintenant souligner une seconde composante. L'équipe était soutenue par un binôme que je constituais avec Paul Aïm ${ }^{3}$ : lui était « le responsable du dedans », celui qui a la plus grande vigilance de l'institution, qui assure la relation à l'école, dans sa complexité ; j'étais « le responsable du dehors ", c'est-à-dire du projet. Sans intelligence commune, il est impossible de tenir la frontière dedans/dehors : la souveraineté de l'institution est telle qu'on ne peut prétendre travailler le dehors sans force réelle à l'intérieur.

Mais nous avons connu les deux expériences. Les premiers projets (San Francisco, Haïti) se sont effectivement faits contre l'institution. Elle s'opposait à la fois au voyage à l'étranger et à ce que les normaliens soient responsables de projets d'école en France ; nous en faisions des acteurs, des moteurs; c'était les faire sortir de leur rôle. Le directeur de l'École normale, l'inspecteur d'académie de l'époque s'opposaient au projet en arguant que nos méthodes n'étaient pas comprises par le milieu enseignant, en particulier le Syndicat national des instituteurs (le SGEN-CFDT y était favorable). C'est au niveau du ministère que le projet a été soutenu. C'est une constante que j'ai pu 
vérifier en travaillant depuis sur la sociologie de l'école au XIX ${ }^{\mathrm{e}}$ siècle : au sommet de l'État, une conscience géopolitique permet de soutenir un travail qui au niveau local est perçu comme une incongruité, une fantaisie dérangeante. C'est vrai de l'international comme de tout ce qui est « le dehors » de l'école, tout ce qui n'est pas dans le consensus dogmatique. Ceci dit, à partir du moment où des enseignants, des formateurs s'engagent avec conviction, et dans la durée, l'institution est capable d'évoluer. Nous avons fait ensuite un projet avec le Maghreb sans aucune difficulté institutionnelle. Ensuite, à partir de 1985-1986, avec l'arrivée d'un nouveau directeur à l'École normale et pendant quatre ans, jusqu'en 1988-1989, avec l'arrivée d'un nouveau recteur, le projet «Enjeux éducatifs de la construction européenne» a reçu un appui incontestable. Le recteur présidait le comité d'organisation du Forum « 92-92 » (Hautsde-Seine/Europe).

CHANTAL PAISANT : C'est en 1989 qu'a lieu ce Forum baptisé «92-92» qui a réuni les maîtres engagés dans les projets, les partenaires étrangers, les normaliens : il marque à la fois un aboutissement et un nouveau point de départ pour les projets. La dynamique des deux évolutions que vous décriviez suppose une formation des maîtres titulaires. Comment ont-ils été impliqués?

Daniel Denis : Au début des années 1980, nous cherchions des maîtres volontaires pour participer aux projets, maîtres formateurs enseignant dans les classes d'application ou non (ce qui heurtait l'institution, qui a ses «bons» maitres). Quand le projet de formation internationale a eu une légitimité institutionnelle, on a procédé à un appel d'offre auprès des enseignants prêts à s'engager, au moins deux ans, autour d'un thème défini par eux et qui donnerait lieu à des voyages, des correspondances, des échanges de travaux avec la classe partenaire. L'inspecteur d'académie présidait la commission de sélection des candidatures. Le Forum de 1989 ayant permis d'officialiser un réseau de base, ironiquement baptisé "Erasminus", le choix était donné aux enseignants intéressés de proposer un thème pouvant être traité avec l'un des partenaires du réseau ou de proposer un échange sur la base de leurs contacts personnels à l'étranger, ce qui élargissait le réseau. Toute l'année 1989-1990 a été consacrée à des séminaires de formation aux échanges scolaires et à un stage de formation continue de deux semaines : une semaine pour formaliser un projet, une semaine de stage à l'étranger pour négocier le projet avec le partenaire.

Chantal paIsant : L'organisation du Forum, ces stages, les séjours d'étude des normaliens à l'étranger ne se font pas sans budget. Quelles étaient les sources de financement?

Daniel Denis : Le Conseil général des Hauts-de-Seine a apporté un soutien considérable et durable. Pour le Forum de 1989, le ministère des affaires étrangères a pris en charge le voyage des étrangers en France, le ministère de l'éducation nationale, à travers la Direction des écoles et la DAGIC ${ }^{4}$ a également apporté sa contribution. À partir du moment où le projet devenait officiel, nous avons eu plus d'argent que nous n'en demandions.

CHANTAL PAISANT : Revenons aux normaliens. Vous avez défini deux principes de base, complémentaires de la formation au métier : la dimension interdisciplinaire du projet, son articulation avec la pratique professionnelle. Plus concrètement, comment le projet est-il géré sur les deux ans ? Comment en assurez-vous l'appropriation individuelle et collective?

Daniel Denis : Une même promotion de normaliens pouvait se subdiviser en un certain nombre de filières, ayant chacune sa coloration particulière, son mode d'organisation et où les étudiants travaillaient ensemble pendant deux ans. Quand nous avons été 
reconnus par l'institution, la cooptation n'était plus «sauvage »: en début d'année, notre équipe faisait une offre de formation à «la coopération éducative internationale ». Les candidats à cette filière se regroupaient et avaient un délai de quatre à cinq mois pour renégocier le thème général proposé et définir, chacun, leur projet personnel dans le cadre général. Un thème aussi vaste que « les enjeux éducatifs de la construction européenne» est inaccessible. Il peut se décomposer en autant de thèmes que de participants.

Nous favorisons les petites équipes, de deux ou trois : les uns travaillant sur la cantine, les repas en Angleterre, en Allemagne et en France, les autres sur les méthodes et les rythmes d'apprentissage de la lecture, d'autres enfin sur les programmes d'histoire ou sur les sports à l'école, par exemple. La légitimité de l'objet était discutée avec le formateur responsable qui signait un contrat avec le normalien. Le contrat était double, impliquant d'une part un travail de type didactique ou social en France, sur le terrain, puis en stage à l'étranger et, d'autre part, une soutenance publique de ce travail face aux instituteurs et aux formateurs de l'École normale. Le choix des documents supports de l'exposé (textes, montage vidéo) devait être pertinent par rapport à l'objet retenu.

Chantal paisant : Cela demande un travail suivi dans les classes. Comment le projet se situe-t-il dans le dispositif général des stages pratiques?

Daniel Denis : De façon générale, le normalien allait chaque semaine dans l'école (et ensuite dans la classe) correspondant à son projet. Mais il faut distinguer ici les deux types de stages proposés par l'institution : le stage en responsabilité, épreuve majeure de la formation, était la règle de "droit commun", le projet international ne le perturbant pas. C'est parce que nous avons toujours respecté ce principe que l'institution a pu intégrer le projet international. Il faut aussi être conscient des limites : les normaliens qui suivaient notre formation n'étaient pas ipso facto de bons instituteurs. Par contre, notre projet faisait exploser le stage en tutelle (où le normalien fait la classe en présence du maître titulaire) ; le budget était redistribué en fonction du projet.

Donc, d'un côté, le respect de l'épreuve du réel était affirmé ; de l'autre, l'épreuve de la simulation était ciblée sur un thème précis défini par le normalien ou sur le projet de classe défini par le maître. Les stages en tutelle étaient axés sur le projet et personnalisés et c'est dans ce cadre que les soutenances publiques prenaient sens.

Chantal PAISANT: C'est aussi dans ce cadre que les normaliens pouvaient être directement associés à la préparation du Forum de 1989...

Daniel Denis : L'idée en fait est venue d'eux. Mais il faut préciser notre stratégie : une fois l'offre faite, en début de formation, elle consistait à ne plus parler du projet international. Mon coéquipier, "responsable du dedans ", entraînait le groupe dans l'école, le métier, laissait l'institution l'absorber, jusqu'à ce qu'il cherche le dehors. Vers le mois de novembre commençait la phase de questionnement, de mise en cause, de contre-propositions, de reformulation de ce qui serait "leur» projet. L'année précédant le Forum, j'avais proposé pour thème : «la complexité européenne vue à travers le tournoi des cinq nations ». Délaissant le rugby et ses enjeux sociaux et culturels, les normaliens ont voulu faire un " tournoi des douze ». C'est ainsi qu'ils sont devenus les coorganisateurs de ce qui allait être le «Forum 92-92 » et en ont défini les thèmes avec les instituteurs. La recherche des financements a fait partie de la formation. 
Les appels d'offre et les séminaires d'instituteurs ont suivi; les échanges scolaires «Erasminus » ont été lancés et, avec une nouvelle promotion de normaliens, en 1991-1992, la même stratégie déboucha sur l'idée d'organiser cette fois une rencontre entre les enfants (quarante classes de vingt-cinq élèves), pendant une semaine, dans les Landes. Nous étions allés repérer le site de ce qui serait « la Cité des enfants ", une cité dont les enfants seraient les citoyens. Mais ce projet, contrairement au précédent, n'a pas abouti.

Chantal PaISANT: 1991-1992, c'est aussi l'année de généralisation de la réforme des IUFM, où vous-même et votre équipe renoncez définitivement à ce type de formation. Des adaptations n'étaient-elles pas concevables dans le nouveau contexte?

Daniel Denis : La transformation des Écoles normales en IUFM nous faisait perdre, au milieu du gué, l'autonomie financière et décisionnelle. C'est un premier handicap qui nous enlevait une partie de notre crédibilité auprès des collectivités locales et des écoles. À cela s'ajoutaient des incompréhensions sur les objectifs poursuivis entre la conception de programmes au premier degré, au second et à l'université. Mais la cause majeure qui fait que nous devions mettre un terme à cette aventure est la structure même de la formation coupée au milieu par un concours. Le travail sur projet, en tant que formation au métier, dans la pratique, et avec une ambition de transformation, a besoin de deux ans minimum pour être assumé effectivement - alors qu'aujourd'hui, la formation au métier se fait en un an. L'ironie du sort est que la dissolution du projet international soit intervenue au moment où le traité de Maastricht faisait l'objet d'un examen public et suscitait de fortes résistances culturelles. Les oppositions pour/contre Maastricht se sont faites sur des archétypes qui montrent à quel point le travail dans lequel nous étions engagés était nécessaire socialement. Tout indique qu'il l'est chaque jour davantage.

CHANTAL PAISANT: Dans l'état actuel des choses, où et comment développer des formations à la coopération éducative?

Daniel Denis : Mon choix personnel depuis deux ans a été de prendre de la distance en entreprenant une recherche universitaire sur l'histoire des relations entre les projets de réformes scolaires et les enjeux géopolitiques, aux $\mathrm{XIX}^{\mathrm{e}}$ et $\mathrm{XX}^{\mathrm{e}}$ siècles. C'est un travail passionnant qui montre l'ancienneté et la complexité d'un rapport méconnu entre « éducation nationale » et rivalités internationales. Cela est vrai à tous les niveaux, même celui des méthodes pédagogiques où, suivant leurs tendances, les groupes de pression ont cherché à imiter ce qui se faisait en Angleterre et en Allemagne par exemple, à la fin du XIX ${ }^{\mathrm{e}}$ siècle. L'intérêt de ces connaissances conforte l'idée de mettre en place une filière de formation professionnelle qui ne serait plus stricto sensu une formation d'instituteurs ou de professeurs mais une formation à un nouveau métier, au métier de la médiation interculturelle, de la géopolitique éducative.

Chantal paisant : Est-ce qu'il ne s'agit pas, par définition, d'une composante du métier d'enseignant, plutôt qu'un métier à part entière?

Daniel Denis : Nos douze années d'expérience montrent où était notre idéal, mais dans les conditions de formation actuelles on ne pourra pas faire beaucoup plus qu'une sensibilisation, une initiation aux problèmes internationaux et aux institutions, c'est-àdire un genre d'instruction civique qui ne constitue pas un outil de formation en profondeur. Il faut donc aller vers un métier à part entière car la formation aux échanges internationaux se fait dans des situations d'échanges, conduits dans la durée, avec des classes, avec des groupes d'enfants et d'adolescents. La nouvelle filière de 
formation à laquelle je pense, et où les IUFM auraient un rôle majeur à jouer, pourrait concerner des enseignants en formation continue, des conseillers d'éducation, des animateurs socio-culturels et tous ceux qui ont une expérience des échanges internationaux dans le domaine du sport, ou des séjours linguistiques, par exemple. C'est un nouveau métier à l'articulation du « dedans » et du « dehors », qui supposerait une culture des implications des relations internationales dans les sociétés et faciliterait les relations entre l'école et le hors l'école. C'est là également que le problème linguistique trouvera sa solution et non pas uniquement à l'intérieur de l'école.

Les États concèdent aujourd'hui de nombreux transferts de souveraineté. Cela se fait dans un temps court. En revanche, la construction des citoyennetés, dans cet espace nouveau de l'interculturel, prend beaucoup de temps et cela requiert des dispositifs originaux. L'école ne doit pas se complaire dans une fonction de refuge pour échapper au mouvement qui efface les repères. Elle doit contribuer activement à forger de nouveaux repères. L'Europe n'est pas une discipline à apprendre mais un projet à inventer.

\section{NOTES}

1. L'expérience présentée ici par Daniel Denis s'est déroulée dans la période antérieure à la mise en place des Instituts universitaires de formation des maîtres (IUFM, 1991-1992), ce qui explique les dénominations d'« instituteurs » et d'«Écoles normales » (NDLR).

2. Élèves-instituteurs des anciennes Écoles normales

3. Professeur de philosophie, formateur à l'école normale puis à l'IUFM de Versailles (site d'Antony).

4. Direction des affaires générales, internationales et de coopération.

\section{RÉSUMÉS}

Dans cet entretien, Daniel Denis fait le bilan de douze années d'expérience de formation à la coopération éducative en École normale et en analyse les conditions institutionnelles et pédagogiques. L'idée que la formation aux échanges scolaires ne peut se faire que dans des situations d'échanges conduits dans la durée et avec des classes débouche sur le projet d'une nouvelle filière de formation au métier de la médiation interculturelle et de la géopolitique éducative. 
INDEX

Mots-clés : coopération internationale, échange international, formation des enseignants, échange scolaire

Index géographique : France

\section{AUTEUR}

DANIEL DENIS

Maîtres de conférences, IUFM de Versailles et à l'université Paris-Sud, France. 